\title{
Delay Induced Regular Spike Synchronization in Two Coupled Chaotic Light-Emitting Diode Systems
}

\author{
Xingpan Cui ${ }^{1}$, Yanqiu Che ${ }^{1, \text { a }}$, Bei Liu $^{1}$, Chunxiao Han $^{1}$ \\ ${ }^{1}$ Tianjin Key Laboratory of Information Sensing \& Intelligent Control, Tianjin University of Technology \\ and Education, Tianjin, 300222, China \\ ayqche@tju.edu.cn
}

Keywords: Chaos synchronization; Light-emitting diode; Time delay; Spike synchronization.

\begin{abstract}
We numerically investigate the effects of coupling delays on the synchronization dynamics of two coupled chaotic light-emitting diodes (LEDs) with ac-coupled nonlinear optoelectronic feedback. With zero-lag or very small time-delay coupling, as the coupling strength increases, the coupled system exhibits weak anti-phase multi-spike synchronization, strong anti-phase low-frequency spike synchronization and finally complete chaos synchronization. With a coupling delay large enough, not only the chaotic spiking is eliminated, but also new high amplitude high frequency regular spike synchronization of coupled LEDs can be obtained.
\end{abstract}

\section{Introduction}

Synchronization of coupled nonlinear oscillators is a fundamental phenomenon in nature that has attracted a lot of attention in the last decades [1, 2]. In a general sense, different kinds of synchronization phenomena, including complete synchronization [3], phase synchronization [4], and lag synchronization [5], have been observed and studied.

Light-emitting diodes (LEDs) become more and more popular and have already been embedded into many consumer devices due to their high luminance and low-cost. LEDs are ideal candidates for the implementation of a complex network on a chip. LEDs also provide the foundation for networking using the visible light as communication medium. These networks require fast and stable synchronization. Efficient synchronization is a key aspect of LED networks.

Recently, a GaAs light-emitting diode (LED) with ac-coupled nonlinear optoelectronic feedback has been shown to exhibit complex dynamics [6]. Compared to the semiconductor laser, LEDs display the same dynamics and are much more easily controllable. However, irregular and chaotic spiking sequences observed in a large range of parameters [6, 7], prevents the establishment of stable regular spike synchronization in a LED network. On the other hand, since a finite time delay always exists for signal transmission in coupled systems, it's necessary to take it into account when investigating the synchronization dynamics of coupled nonlinear oscillators. Nowadays, the synchronization dynamics of two coupled chaotic LED system has not been fully understood, especially, how the coupling delay affects the synchronization dynamics of LED networks has not been studied. The goal of this paper is to investigate the effects of coupling delays on the synchronization between two chaotic LED systems. Simulation results demonstrate that, by increasing the coupling delay, one can obtain a new kind of high amplitude high frequency regular spike synchronization in two coupled chaotic LEDs.

\section{LED System Model and Its Complex Dynamics}

For numerical and analytical purposes, the LED system dynamics is written in dimensionless form (see [6] for details):

$$
\dot{x}=x(y-1), \dot{y}=\gamma\left(\delta_{0}-y+\alpha(w+x) /(1+s(w+x))-x y\right), \dot{w}=-\varepsilon(w+x)
$$


where $x$ and $y$ are suitably normalized photon and population-inversion densities and $w$ describes the nonlinear ac feedback loop. $\gamma$ is the ratio between photon and carrier lifetimes. $\delta_{0}$ is the relative bias voltage parameter, $\alpha$ is the feedback strength. $\gamma$ and $\varepsilon$ are time-scale parameters. In this paper we set the parameter values as $s=0.2, \alpha=1.002, \gamma=3.3 \times 10^{-3}$, and $\varepsilon=4 \times 10^{-5}$.

Figure 1 shows a detailed bifurcation diagram computed from Eq. (1) by varying $\delta_{0}$ over a small interval contiguous to the initial Hopf bifurcation. The system passes through a cascade of period doubled and chaotic attractors. A period doubling route to chaos occurs.

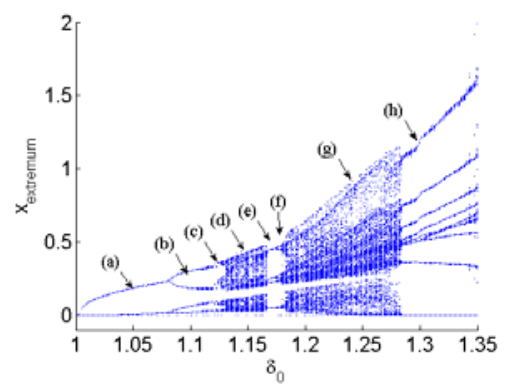

Figure 1. Bifurcation diagram for the peak values of $x$ as the parameter $\delta_{0}$ is varied.

Now we couple two LED systems bidirectionally through the bias voltage with coupling time delay. The corresponding model is

$$
\begin{aligned}
& \dot{x}_{1}=x_{1}\left(y_{1}-1\right), \dot{y}_{1}=\gamma\left(\delta_{0}-y_{1}+\alpha w_{1}+x_{1} /\left(1+s\left(w_{1}+x_{1}\right)\right)-x_{1} y_{1}\right)+g_{c}\left(y_{2}(t-\tau)-y_{1}(t)\right), \dot{w}_{1}=-\varepsilon\left(w_{1}+x_{1}\right) \\
& \dot{x}_{2}=x_{2}\left(y_{2}-1\right), \dot{y}_{2}=\gamma\left(\delta_{0}-y_{2}+\alpha\left(w_{2}+x_{2}\right) /\left(1+s\left(w_{2}+x_{2}\right)\right)-x_{2} y_{2}\right)+g_{c}\left(y_{1}(t-\tau)-y_{2}(t)\right), \dot{w}_{2}=-\varepsilon\left(w_{2}+x_{2}\right)
\end{aligned}
$$

where the subscripts 1 and 2 indicate two LED systems, and are coupling strength and delay time between those two LEDs.

In this paper, we only consider the case of two identical LEDs in chaotic regime $\left(\delta_{0}=1.15\right)$ and investigate the effects of $g_{c}$ and $\tau$ on the synchronization dynamics.

\section{Simulation Results and Analysis}

In this section, we first study the synchronization dynamics of coupled chaotic LEDs without coupling delay. Figure 2 shows the spike frequency (Fig. 2(a)) and amplitude (Fig. 2(b)) as a function of coupling strength. The dashed black lines separate the parameter space into three different regimes in term of the synchronization dynamics.
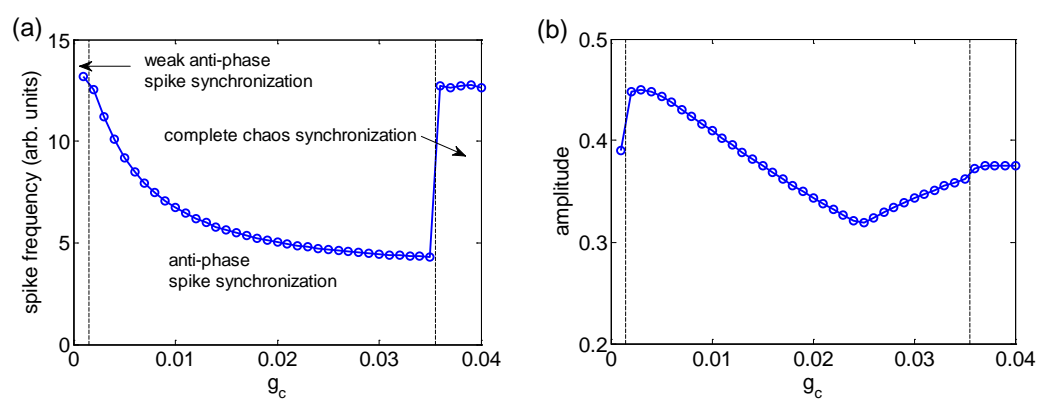

Figure 2: Bifurcation diagram of spike frequency and amplitude as a function of coupling strength without delay. (a) frequency (b) amplitude.

Figure 3 gives typical responses of time series of states $x_{1}$ and $x_{4}$ (Fig. 3(a)) and the corresponding phase portraits on $x_{1}-x_{4}$ plans (Fig. 3(b)). For a very small coupling strength ( $g_{c}=0.001$ ), weak anti-phase multi-spike synchronization between the two chaotic LEDs is observed. For a mediate coupling strength $\left(g_{c}=0.005,0.025,0.035\right)$, one can obtain anti-phase low frequency 
spike synchronization with a decreasing frequency and different amplitudes. For a large coupling strength $\left(g_{c}=0.04\right)$, the dynamics becomes complete chaotic synchronization. As we increase the coupling strength, we find transitions from weak anti-phase multi-spike synchronization, to anti-phase low frequency spike synchronization, to complete chaos synchronization.

(a)
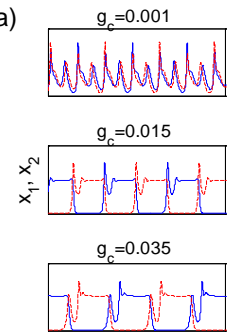
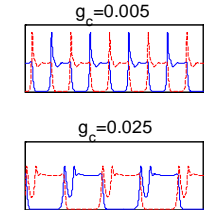

$g_{c}=0.04$

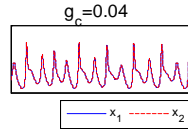

(b)
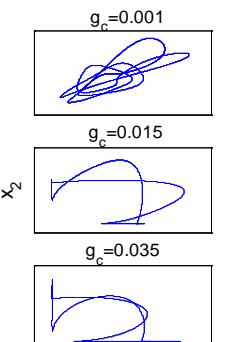
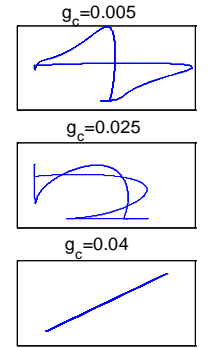

Figure 3: Responses of the coupled LEDs with different coupling strengths: (a) time series of $x_{1}, x_{2}$, (b) the corresponding phase portraits on $x_{1}-x_{2}$ plane.

Next, we take into account the coupling time delays and investigate how it affects the above transitions. Figure 4 gives the bifurcation diagram of spike frequency (Fig. 4(a)) and amplitude (Fig. 4(b)) as a function of coupling strength with different coupling delay. Figure 5 shows typical responses of time series of states $x_{1}$ and $x_{4}$ (Fig. 5(a)) and the corresponding phase portraits on $x_{1}-x_{4}$ planes (Fig. 5(b)) with different parameter values. For small coupling delay (Fig. 4 and 5, $\tau=10$ ), the delay doesn't change the synchronization dynamics and their transitions qualitatively. When the coupling delay is big enough (Fig. 4 and 5, $\tau=30,50,70,90$ ), in the regime of anti-phase low frequency spike synchronization (mediate coupling strengths), the spike frequencies are almost the same, while the amplitude becomes larger as the coupling delay increases. The bifurcation values of $g_{c}$ for the transitions (Fig. 4, sudden increases of frequency and amplitude) from anti-phase low frequency spike synchronization to complete chaos synchronization $(\tau=10)$ or to complete regular spike synchronization ( $\tau=30,50,70,90)$ decreases as $\tau$ increases. When $\tau$ exceeds a certain threshold value, high amplitude and high frequency spike synchronization occurs.

(a)

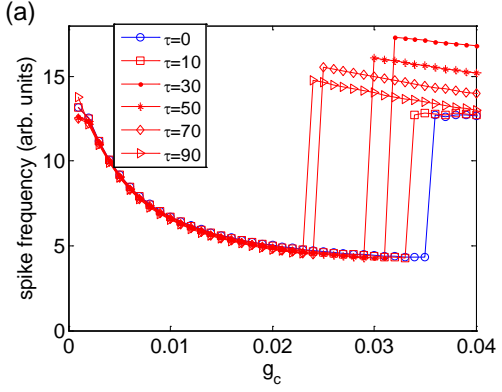

(b)

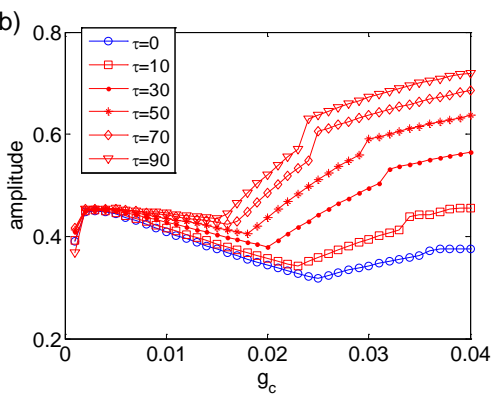

Figure 4: Bifurcation diagram of spike frequency and amplitude as a function of coupling strength with different coupling delays. (a) frequency (b) amplitude.

\section{Summary}

In any physical systems, time delays are unavoidable in signal transmission. In this paper, we have numerically studied the effects of coupling delays on the synchronization dynamics between two LEDs in chaotic regime. We have shown that without the coupling delay, with increase of coupling strength, the coupled system undergoes transitions from weak anti-phase chaotic synchronization, to anti-phase chaotic synchronization, to anti-phase low-frequency spiking synchronization and finally to chaotic synchronization. On the other hand, for a coupling strength large enough, as the coupling 
delay exceeds a certain critical value, a new dynamics, i.e. high amplitude high-frequency spiking synchronization occurs. The systems fire more regularly. In conclusion, we have uncovered that time delay facilitates regular spike synchronization between two coupled chaotic LEDs, which is useful for real-world applications. The effects of coupling delays on synchronization of complex network with more than two interacting LEDs will be our next step of study.

(a)
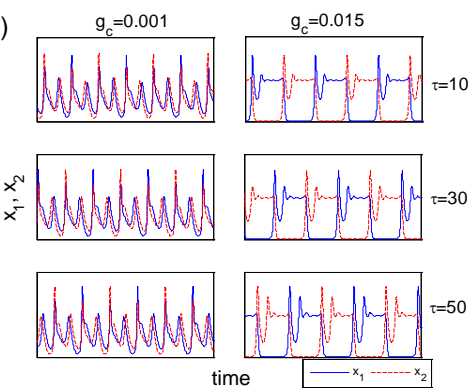

(c)

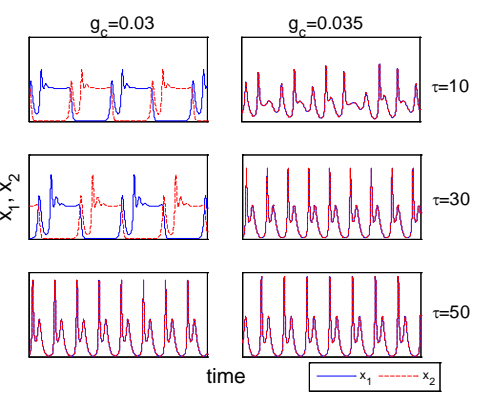

(b)
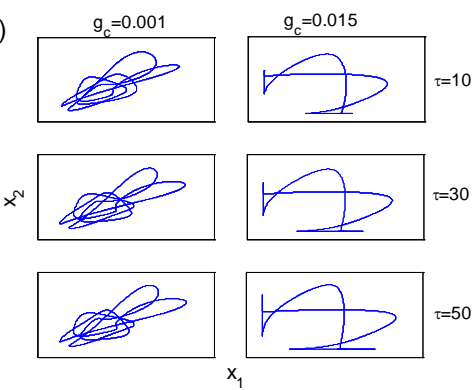

(d)
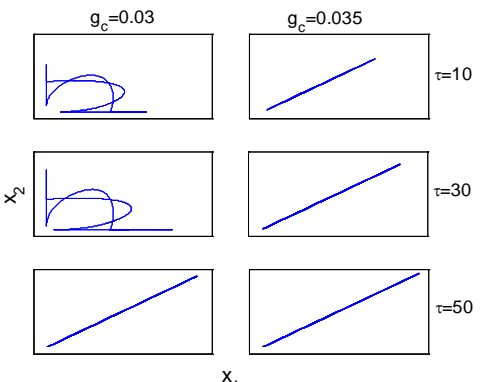

Figure 5: Example responses of the coupled LEDs with different coupling strengths and delays: $(\mathrm{a}, \mathrm{c})$ time series of $x_{1}, x_{2},(b, d)$ the corresponding phase portraits on $x_{1}-x_{2}$ plane.

\section{Acknowledgments}

The National Natural Science Foundation of China (Grants No. 61178081, and No. 61104032). We would also acknowledge the support of Tianjin University of Technology and Education (Grants Nos: RC14-09, RC14-49 and RC14-59).

\section{References}

[1] J. Kurths, A. Pikovsky and M. Rosenblum, Synchronization: a universal concept in nonlinear science (University Press, Cambridge: Cambridge, 2001)

[2] C. M. Gray, P. König, A. K. Engel, and W. Singer, Nature (London) Vol. 338 (1989), p. 334.

[3] L.M. Pecora and T.L. Carroll: Phys. Rev. Lett. Vol. 64 (1990) p. 821.

[4] M.G. Rosenblum, A.S. Pikovsky, and J. Kurths: Phys. Rev. Lett. Vol. 76 (1996), p. 1084.

[5] J.W. Shuai, D.M. Durand, Phase synchronization in two coupled chaotic neurons, Phys. Lett. A 264 (1999) 289-297.

[6] F. Marino, M. Ciszak, S.F. Abdalah, K. Al-Naimee, R. Meucci and F.T. Arecchi: Phys. Rev. E Vol. 84 (2011), p. 047201.

[7] R.M. Sanner and J.E. Slotine Gaussian networks for direct adaptive control. IEEE Trans Neural Network Vol. 3 (1992), p. 837. K. Al-Naimee, F. Marino, M. Ciszak, R. Meucci, and F. T. Arecchi: New J. Phys. Vol. 11 (2009), p. 1-11. 